\title{
TECHNIQUE DETERMINANTS OF KNEE ABDUCTION MOMENTS DURING PIVOTING IN FEMALE SOCCER PLAYERS.
}

Paul A. Jones ${ }^{\mathrm{a}}$, Lee C. Herrington ${ }^{\mathrm{a}}$ and Philip Graham-Smith ${ }^{\mathrm{a}, \mathrm{b}}$

${ }^{a}$ Directorate of Sport, Exercise and Physiotherapy, University of Salford.

${ }^{\mathrm{b}}$ Aspire Academy, Doha, Qatar.

Corresponding Author: Dr. Paul Jones. Directorate of Sport, Exercise and Physiotherapy, University of Salford, Allerton Building, Frederick Road Campus, Salford, Greater Manchester, United Kingdom, M6 6PU.

Tel: (+44) 161295 2371. Email: P.A.Jones@ salford.ac.uk

Additional Author contact details:

Dr. Lee Herrington. Directorate of Sport, Exercise and Physiotherapy, University of Salford, Allerton Building, Frederick Road Campus, Salford, Greater Manchester, United Kingdom, M6 6PU.

Email: $\underline{\text { L.C.Herrington@salford.ac.uk }}$

Dr. Philip Graham-Smith*, Aspire Academy, PO BOX 22287, Doha, Qatar.

Email: Philip.GrahamSmith@aspire.qa

*This is the authors' current address, but during data collection and analysis the author was at the University of Salford.

Abstract: 202 words

Word Count: 4168 words 


\section{ABSTRACT}

2 Background: No previous studies have investigated the optimal technique for pivoting with

3 regard to reducing peak knee abduction moments and potential knee injury risk. The aim of

4 this study was to investigate the relationships between technique characteristics and peak knee

5 abduction moments during pivoting.

6 Methods: Twenty-seven female soccer players [mean (SD); age: 21 (3.8) years, height: 1.67

7 (0.07) m, and mass: $60.0(7.2) \mathrm{kg}$ ] participated in the study. Three dimensional motion

8 analyses of pivots on the right leg were performed using 10 Qualysis 'Pro reflex' infrared

9 cameras $(240 \mathrm{~Hz})$. Ground reaction forces were collected from two AMTI force platforms

$10(1200 \mathrm{~Hz})$ embedded into the running track to examine penultimate and final contact.

11 Pearson's correlation coefficients, co-efficients of determination and stepwise multiple regression were used to explore relationships between a range of technique parameters and peak knee abduction moments. Significance was set at $\mathrm{P}<0.05$.

Findings: Stepwise multiple regression found that initial foot progression and initial knee abduction angles together could explain 35\% (30\% adjusted) of the variation in peak knee abduction moments $\left(\mathrm{F}_{(2,26)}=6.499, \mathrm{P}=0.006\right)$.

Interpretation: The results of the present study suggest that initial- foot progression and knee abduction angles are potential technique factors to lower knee abduction moments during pivoting.

Keywords: Anterior Cruciate Ligament; Injury; Knee Abduction Moment; Technique; $180^{\circ}$ Turns 


\subsection{INTRODUCTION}

Cutting and pivoting have been identified as key actions associated with non-contact anterior cruciate ligament (ACL) injuries in female athletes (Boden, Dean, Feagin \& Garrett, 2000; Olsen, Myklebust, Engebretsen \& Bahr, 2004; Faude, Junge, Kindermann \& Dvorak 2005), as such actions involve lower limb postures that increase knee abduction moments (Cortes et al., 2011), which could lead to increased ACL strain (Shin, Chaudhari, \& Andriacchi, 2009; Shin, Chaudhari, \& Andriacchi, 2011) and subsequent injury. Several studies have investigated optimal cutting technique for reducing knee abduction moments and knee injury risk (McLean, Huang \& van der Bogert, 2005; Sigward \& Powers, 2007; Dempsey, Lloyd, Elliot, Steele, Munro \& Russo, 2007; Dempsey, Lloyd, Elliot, Steele \& Munro, 2009; Jamison, Pan \& Chaudhari, 2012; Kristianlunds, Faul, Bahr, Myklebust \& Krosshaug, 2014; Havens \& Sigward, 2015; Jones, Herrington \& Graham-Smith, 2015), whilst no previous studies have examined pivoting or $180^{\circ}$ turns in this regard.

Previous research into cutting has revealed that the magnitude of lateral leg plant (Dempsey et al., 2007; Dempsey et al., 2009; Havens \& Sigward, 2015; Jones et al., 2015), lateral trunk flexion (Dempsey et al., 2007; Dempsey et al., 2009; Jamison et al., 2012; Jones et al., 2015) and initial knee abduction angles (McLean et al., 2005; Kristianlunds et al., 2014; Jones et al., 2015) are influential in determining the magnitude of peak knee abduction moments. McLean et al. (2005) examined initial lower limb postures in 10 male and 10 female NCAA athletes performing $45^{\circ}$ side-step cuts and found greater peak knee abduction moments were associated with larger initial hip flexion, internal rotation and knee abduction angles, with knee abduction moments more sensitive to the later 2 variables in females. In addition, Sigward and Powers (2007) found that lateral ground reaction forces (GRF), initialfoot progression, hip rotation and abduction angles could explain $49 \%$ of the variation in peak knee abduction moments during $45^{\circ}$ cutting in female soccer players. Such technique aspects 
are a likely result of performance demands. For example, a wide lateral foot placement during cutting is necessary to generate medial GRF to facilitate the direction change.

As mentioned previously, a limitation of previous studies into optimal cutting technique for injury prevention is that with the exception of a few (Kristianlunds et al., 2014; Havens \& Sigward, 2015; Jones et al., 2015), the majority of studies have only considered cutting between the angles of 30 to $60^{\circ}$, whilst none have examined pivoting $\left(180^{\circ}\right)$. Notational analysis in male Premier league soccer has shown that changing direction manoeuvres involving greater angles of direction change $\left(90\right.$ to $\left.180^{\circ}\right)($ Bloomfield, Polman \& O’Donoghue, 2007) can frequently occur, and these may exacerbate knee joint loads. Cortes et al. (2011) found that pivoting significantly increases knee abduction motion and moments $\left[-12.2(7.0)^{0} / 0.72(0.3)\right.$ N.m/kg.m] compared to drop jump landings [-3.9 (8.0) $\%$ 0.14 (0.07) N.m/kg.m] and $45^{\circ}$ cutting [-3.8 $(10)^{\circ} / 0.17(0.5)$ N.m/kg.m] in female soccer players. This is perhaps due to the different task demands, with the need to decelerate to a complete stop before accelerating again during the pivot compared to laterally planting the leg and shifting momentum to the opposite side during a $45^{\circ}$ cut.

Because of the different task demands between cutting and pivoting many of the parameters previously found with regard to optimal cutting technique may not necessarily be associated with peak knee abduction moments during pivoting. However, some of the variables identified previously such as initial knee abduction (McLean et al., 2005; Kristianlunds et al., 2014; Jones et al., 2015), hip internal rotation angles (McLean et al., 2005; Sigward and Powers, 2007; Havens \& Sigward, 2015) and lateral trunk flexion (Dempsey et al., 2007; Dempsey et al., 2009; Jamison et al., 2012; Jones et al., 2015) might be expected to be associated with peak knee abduction moments during pivoting. Increased initial hip internal rotation angles leads to a more medially placed knee (i.e., greater initial knee abduction angle) relative to the GRF vector, resulting in an increased moment arm that 
would elevate knee abduction moments during changing direction tasks (Sigward \& Powers, 2007). Whereas trunk position during landing and changing direction manoeuvres is often a critical factor in influencing knee joint loads (Mendiguchia et al., 2011) as the trunk is the largest segment of the body and thus, influences the position of the GRF vector relative to the knee joint during such manoeuvres. Therefore, initial knee abduction, hip rotation, and sagittal and frontal plane trunk flexion may influence knee abduction moments during pivoting and thus, should be considered in developing a model of technique for this manoeuvre.

Previous research (Cortes et al., 2011) has suggested that increased initial foot progression angle away from the direction of travel may account for the high knee abduction moments observed during pivoting. An increased initial foot progression angle or a more rotated pelvis during pivoting would be an attempt by athletes to facilitate the direction change by reducing the amount of rotation required during final contact (the phase when a subject makes contact with the ground and initiates movement into a different direction) and then re-acceleration. However, greater initial foot progression angle (or pelvic rotation) would lead to athletes absorbing the large impact forces at final contact through the frontal plane potentially increasing knee abduction moments, whereas reducing this angle would allow the large forces to be absorbed through the sagittal plane utilising the large knee and hip extensor muscle groups (e.g., peak external knee and hip flexor moments). Furthermore, if the thigh is more abducted or the foot is planted a large distance from the pelvis (i.e., greater last step length or horizontal distance between pelvis and foot) with an increased foot progression angle may further increase the moment arm of the GRF vector relative to the knee joint (similar to the effect of increased lateral leg plant during cutting) and thus increase peak knee abduction moments. Therefore, research into developing an optimal technique for pivoting should investigate these variables to confirm such a hypothesis. 

the opposite direction, whereas cutting involves shifting momentum into a different direction. Therefore, the deceleration strategy during pivoting may be influential in lowering forces during final contact and subsequently knee abduction moments. Graham-Smith, Atkinson, Barlow and Jones (2009) have found that penultimate contact ( $2^{\text {nd }}$ to last foot contact with the ground during a pivot before moving into a new intended direction) prior to the turn resulted in greater vertical and anterior-posterior GRF's and internal knee extensor moments compared to final contact during a pivot in male soccer players. Thus, analysis of penultimate contact may provide more insight into the optimal technique for pivoting for reduced knee injury risk. Theoretically, if the majority of forward momentum can be reduced during penultimate contact, then lower external knee abduction moments may be experienced during the turn, where injuries often occur (Boden et al., 2000; Olsen et al., 2004) due to lower resultant GRF's. If the deceleration strategy is emphasised towards final contact this will increase resultant GRF at final contact which could increase peak knee abduction moments (GrahamSmith et al., 2009; Jones et al., 2015). Research should perhaps consider the deceleration peak horizontal GRF ratio (HGRFR). Thus, if greater horizontal force can be generated during the penultimate contact relative to the final contact (i.e., a lower ratio) this may indicate greater braking during the penultimate contact which may lower resultant GRF and characteristics and peak knee abduction moments during pivoting. The study investigates whether HGRFR, sagittal plane hip and knee joint moments and a number of initial lower limb, pelvis and trunk positions are associated with peak knee abduction moments. It is hypothesised that these variables are related to peak knee abduction moments during pivoting. 
2.0 METHODS

127

\subsection{SUBJECTS}

Twenty-seven female soccer players [mean (SD); age: 21 (3.8) years, height: 1.67 (0.07) m, and mass: $60.0(7.2) \mathrm{kg}$ ] acted as subjects for the study. All players were registered with Soccer clubs playing in the second tier of English Women's Soccer. Written informed consent was attained from all subjects and approval for the study was provided by the University's ethical committee.

\subsection{RESEARCH DESIGN}

Testing took place on an indoor Mondo running surface. Each subject was required to attend the lab on 2 separate occasions. The first occasion was a familiarization session on the protocols used in the study with data collected on the subsequent session. The pivot involved the subjects running towards 2 force platforms. The first force platform was used to measure GRFs from the penultimate (left) foot contact, whilst the $2^{\text {nd }}$ force platform was used to measure GRFs from the final (right) foot contact. Prior to the turn the subject ran through, a set of timing lights $5 \mathrm{~m}$ from the centre of the last platform. The subjects then turned $\left(180^{\circ}\right)$ back to the original starting position once contacting the end force platform with the right leg. Total time to complete the task was measured using a set of Brower timing lights (Draper, UT). The timing lights were set at approximate hip height for all subjects as previously recommended (Yeadon, Kato \& Kerwin, 1999), to ensure that only one body part (i.e., lower torso) breaks the beam. Task completion time was used to monitor performance between trials and subjects. During familiarization and practice trials subjects were given feedback to regulate the time to complete the task, so that they could gage the speed of approach they used during subsequent data collection. Each subject started approximately 5 metres behind the 
first set of timing lights. Some flexibility was allowed for the exact starting point for each subject to allow for the subjects differing stride pattern as they approached the end 2 force platforms. Each subject was allowed time prior to data collection to identify their exact starting point to ensure appropriate force platform contacts.

During data collection all subjects performed a minimum of 6 'Good' trials of the pivot task. A good trial was considered to involve; 1) a straight approach to the force plates without prior stuttering or prematurely turning prior to final contact, 2) contact with the first force platform during penultimate (left) foot contact 3 ) contact with the central portion of the last platform during final contact to ensure a homogeneous distance of travel between trials and 4) recording an appropriate time to complete the task [2.65 s (10\%)]. Trials were subsequently disqualified if the subject did not adhere to these characteristics. Verbal feedback was provided to rectify any of the abovementioned aspects on subsequent trials. The turn times were selected based on pilot work and used to control for performance of the tasks within and between subjects. In addition, for each trial the horizontal velocity in the direction of motion of the right hip joint centre was calculated over the 10 frames prior to penultimate foot contact to determine approach velocity in accordance with McLean et al. (2005). This retrospective analysis was conducted to ensure that each subjects trial achieved a target approach velocity of between 3.6 to $4.4 \mathrm{~m} \cdot \mathrm{s}^{-1}$ for the pivot task. These target approach velocities were selected based on velocities recorded in several previous studies (McLean et al., 2005; Cotes et al., 2011) and previous pilot data collected in this lab. 


\subsection{PROCEDURES}

The procedures have been reported previously (Jones et al., 2014; Jones et al., 2015).

Thus, only a brief overview is provided here. Reflective markers (14 $\mathrm{mm}$ spheres) were placed on body landmarks (see Jones et al., 2014) of each subject by the same researcher to ensure marker placement consistency. Subjects wore 'cluster sets' (4 reflective markers attached to a light weight rigid plastic shell) attached using Velcro elasticated wraps on the right and left thigh and shin to approximate the motion of these segments during dynamic trials. The pelvis and trunk cluster sets were attached using an elasticated belt and Lycra 'crop top', respectively.

Three dimensional motions of these markers were collected whilst performing the pivots using 10 Qualysis 'Pro reflex' (Model no. MCU 240) infrared cameras (240Hz) operating through Qualysis Track Manager software (version 1.10.282). GRFs were collected from two AMTI (Model no. 600900) force platforms (1200Hz) embedded into the running track. The force platform arrangement allowed data to be collected for both the final and penultimate contact.

From a standing trial, a 6-degree-of-freedom model of the lower extremity and trunk was created for each participant, including trunk, pelvis, thigh, shank and foot using Visual 3D software (C-motion, version 3.90.21). This kinematic model was used to quantify the motion at the hip, knee and ankle joints using Cardan angle sequence (Grood \& Suntay, 1983). The local coordinate system was defined at the proximal joint centre for each segment. The static trial position was designated as the subject's neutral (anatomical zero) alignment, and subsequent kinematic measures were related back to this position. Lower limb joint moments were calculated using an inverse dynamics approach (Winter, 1990) through Visual3d software (C-motion, version 3.90.21) and are defined as external moments. Segmental inertial characteristics were estimated for each participant (Dempster, 1955). The 
model utilised a CODA pelvis orientation (Bell, Brand \& Pedersen, 1989) to define the location of the hip joint centre. The knee and ankle joint centres were defined as the mid-point of the line between lateral and medial markers. A minimum of 4 trials were used in the analysis of each subject based on visual inspection of the motion files. Trials were disqualified if approach velocity fell outside of the desired ranges stated above or if the subjects slid, turned prematurely or missed the force platform that went unnoticed during data collection. The trials were time normalised for each subject, with respect to the ground contact time of the pivot. Initial contact was defined as the instant after ground contact that the vertical GRF (vGRF) was higher than $20 \mathrm{~N}$ and end of contact was defined as the point where the vGRF subsided past $20 \mathrm{~N}$ for both penultimate and final contacts. The weight acceptance phase of ground contact was defined as from the instant of initial contact (vGRF $>20 \mathrm{~N}$ ) to the point of maximum knee flexion during ground contact as used previously (Havens \& Sigward; 2015; Jones et al., 2015). Joint coordinate and force data were smoothed in visual $3 \mathrm{D}$ with a Butterworth low pass digital filter with cut-off frequencies of $12 \mathrm{~Hz}$ and $25 \mathrm{~Hz}$, respectively. Cut off frequencies were selected based on a residual analysis (Winter, 1990) and visual inspection of the data.

During final contact of the pivot task the following angles were determined at the point of initial contact; foot progression (angle of foot orientation relative to the original direction of travel $\left[0^{\circ}\right.$ straight, positive rotated inward (anti-clockwise), negative rotated outward (clockwise)], pelvic rotation (angle of the pelvis in the transverse plane relative to the original direction of travel at initial contact $\left[0^{\circ}\right.$ neutral pelvis position, positive anticlockwise rotation]), knee abduction (positive adduction/ negative abduction), hip abduction (positive adduction/ negative abduction) and rotation (positive internal rotation/ negative external rotation), hip, knee, and ankle in the sagittal plane, trunk flexion relative to a vertical line perpendicular to the pelvis $\left(0^{\circ}\right.$ upright, positive trunk lean forward, negative trunk leaning 
back) and lateral trunk flexion relative to a vertical line perpendicular to the pelvis $\left(0^{\circ}\right.$ upright, positive trunk lean away from the planted leg, negative trunk leaning towards the

227 planted leg). Touchdown distance (horizontal distance from the centre of mass of the pelvis 228 to centre of mass of the right foot at initial contact using the global co-ordinate system) and 229 last step length (horizontal distance from the centre of mass of the left foot at penultimate contact to the right foot at final contact using the global co-ordinate system), sagittal plane peak knee and hip flexor moments during final contact were also determined. To evaluate deceleration strategy from penultimate to final contact and its relationship to peak knee abduction moments during final contact, a final/ penultimate contact horizontal (Fx component) GRF ratio (HGRFR) was also calculated.

\subsection{STATISTICAL ANALYSIS}

All statistical analysis was performed in SPSS for windows v17 (Chicago, Ill). Normality for each variable was examined using a Shapiro-Wilks test. Pearson's correlation coefficients, co-efficients of determination $\left(\mathrm{R}^{2} \times 100 \%\right)$ and stepwise multiple regression were used to explore relationships of the abovementioned variables and peak knee abduction moments. For the stepwise multiple regression only significantly correlated variables were considered. Significance was set at $\mathrm{P}<0.05$.

\subsection{RESULTS}

Descriptives for each variable can be found in Table 1. Mean (SD) approach velocity and total times to the complete the task were $4.02(0.2) \mathrm{m} \cdot \mathrm{s}^{-1}$ and $2.67(0.11) \mathrm{s}$, respectively. Only initial foot progression (Figure 2b), initial knee abduction angles (Figure 2a) and peak hip flexor moments were significantly $(\mathrm{p}<0.05)$ correlated to peak knee abduction moments 
(Table 1) during final contact. Stepwise multiple regression analysis found that initial foot

251

252

253

254

255

256

257

258

259

260

261

262

263

264

265

266

267

268

269

270

271

272

progression angle and initial knee abduction angle together could explain 35\% (30\% adjusted)

of the variation in peak knee abduction moments $\left(\mathrm{F}_{(2,26)}=6.499, \mathrm{P}=0.006\right)$. The regression equation is summarised in Table 2.

\subsection{DISCUSSION}

The aim of the present study was to investigate the relationships between predetermined technique characteristics and peak knee abduction moments during pivoting. Initial foot progression and knee abduction angles were the main predictors of peak knee abduction moments (35\%) during pivoting, providing support for these variables in the apriori theory.

Previous research (McLean et al., 2005; Sigward \& Powers, 2007; Dempsey et al., 2007; Dempsey et al., 2009), have attempted to evaluate technique characteristics responsible for increasing peak knee abduction moments during 30 to $60^{\circ}$ cutting, which may not truly represent the changing direction demands of soccer (Bloomfield et al., 2007; Greig, 2009). No previous research has examined pivoting with regard to technique determinants of peak knee abduction moments. In the present study, only initial knee abduction and foot progression angles were found to be related to peak knee abduction moments, explaining $35 \%$ (30\% adjusted) of the variation. Cortes et al. (2011) previously suggested that increased (inward) foot progression angle may be a key variable that could influence knee joint loads during pivoting, but presented no data to support this. Reducing the initial foot progression angle to a close to straight foot position, has the effect of allowing the large forces to be absorbed through the sagittal plane utilising the large knee and hip extensor muscle groups to fully absorb the GRF generated. In support of this, a significant correlation was observed between peak hip flexor moments and peak knee abduction moments $\left(R=-0.388, R^{2}=15 \%, P<0.05\right)$. 
The greater the peak hip flexor moments produced during final contact the lower the peak knee abduction moments. Whereas a more rotated foot during weight acceptance creates an external knee abduction moment, as the force vector is lateral to the knee joint. It should be noted however, that in order to then execute the turn from a straighter initial foot position, the athlete should unload to allow the foot to rotate and avoid generating large rotational stress at the shoe-surface interface.

Increased initial knee abduction angle was also found to be significantly related to peak knee abduction moments and has previously been found for cutting (McLean et al., 2005; Kristianlunds et al., 2014; Jones et al., 2015). Greater initial knee abduction angles have the effect of shifting the knee more medial relative to the GRF vector. This in turn leads to a greater moment arm between the knee joint axis and GRF vector and consequently greater knee abduction moments. Therefore, as with cutting it is recommended that during pivoting, athletes avoid or limit the amount of knee abduction during early ground contact to lower knee abduction moments.

Increased maximal horizontal braking forces $[-1.79(0.29) \mathrm{BW}]$ during the penultimate contact relative to the final contact $[-1.65(0.29) \mathrm{BW}]$ were observed; substantiating our earlier research on pivoting in male soccer players (Graham-Smith et al., 2009) and 90 cutting in female soccer players (Jones et al., 2015). Theoretically, this deceleration strategy has the advantage of reducing the resultant GRF during final contact, which would influence external knee joint loads during final contact. When considering the HGRFR for both manoeuvres no relationship was observed with peak knee abduction moments. However, on further analysis players with greater $(n=9)$ peak knee abduction moments $(+0.5 \mathrm{SD})$ had a higher ratio than players exhibiting lower $(n=8)$ peak knee abduction moments $(-0.5$ SD) [0.99 (0.24) vs. $0.92(0.18)]$; similar to our earlier research on $90^{\circ}$ cutting (Jones et al., 2015) and suggests that players with lower peak knee abduction moments do so by braking more 
during penultimate contact. Therefore, the lack of a relationship found may be due to the low sample size in the present study. Future studies should perhaps consider a more in-depth kinetic and kinematic evaluation of the penultimate contact in order to gather a greater understanding of the role of penultimate contact during pivoting and potentially develop a more comprehensive model of optimal technique for the manoeuvre.

A limitation of the present study is the pre-planned execution of the pivot task as opposed to unanticipated, which has been used in previous studies (Besier, Lloyd, Cochrane \& Ackland, 2001; Cortes et al., 2011) and shown to elevate knee joint loads during cutting (Besier et al., 2001). Future studies need to confirm the technique factors identified in the present study under unanticipated conditions.

Another limitation of the present study, is that the model developed only included 2 variables and explained $35 \%$ of the variance in peak knee abduction moments, thus, perhaps limits the application of these findings in developing a model of optimal technique for pivoting to reduce injury risk. This may be due to the generally low sample size used in the present study $(\mathrm{n}=27)$, which limits the number of variables that can be integrated into the model (Vincent, 1995). For instance, a greater sample size may have led to the inclusion of the significantly correlated peak hip flexor moments into the model. Furthermore, it is possible that additional variables have been missed by the authors in the a-priori theory. As mentioned above, some further kinematic and kinetic variables from penultimate contact may be associated with peak knee abduction moments during final contact. Thus, further research particularly of penultimate contact is needed to develop this model further in order to identify a definitive model of technique for pivoting with regard to injury prevention.

Previous research into $45-90^{\circ}$ cutting in males and females have found associations between peak knee abduction moments and initial hip internal rotation (Sigward \& Powers, 2007; Havens \& Sigward, 2015), hip abduction (Sigward \& Powers, 2007), lateral trunk 

it was expected that these variables may be related to peak knee abduction moments during pivoting. With many of these variables showing no or weak correlations $(R \leq 0.3)$ it is unlikely that they are related to peak knee abduction moments during pivoting. Although low, both initial pelvis and hip internal rotation angles revealed correlations greater than 0.3 with the later close to significance $(\mathrm{P}=0.07)$ and are thus, worth considering in future investigations with greater sample sizes to develop a model of technique for pivoting.

Finally, due to the need to control for performance aspects (i.e., turn times, approach velocity) between subjects it was beyond the scope of the study to evaluate what technique aspects influence performance and whether such aspects would contradict the findings from the present study for reducing peak knee abduction moments. For example, an increased foot progression angle might be beneficial for reducing total time to complete the task, as this would help rotate more of the body prior to final foot contact but has the negative effect of increasing peak knee abduction moments. Future research should examine this conflict between performance requirements and injury risk during changing direction tasks in more detail by examining what technique parameters are associated with total time to complete the pivot task used in the present study (i.e., subjects aim to complete the task as fast as possible without controlling for approach velocity and performance time) and whether these parameters are also associated with large peak knee abduction moments. Without recognising the implications for performance in research, limits the application of any findings related to injury prevention through technique interventions during agility training methods, as players and coaches are more likely to adhere to training programmes with a performance centred focus. 
The aim of the present study was to investigate the relationships between technique/

352 biomechanical characteristics and peak knee abduction moments during pivoting. Initial foot

353 progression and knee abduction angles were identified as significant technique predictors of

354 peak knee abduction moments during pivoting. These findings reveal potential technique

355 factors to develop a model for pivoting technique for injury prevention purposes.

356

357 ACKNOWLEDGEMENTS

358 No funding was received to support this study. The authors have no conflict of interest.

359

360

361

362

363

364

365

366

367

368

369

370 


\subsection{REFERENCES}

372 Angeloni, C., Cappozzo, A., Catani, F., \& Leardini, A. (1993). Quantification of relative 373 displacement of skin- and plate- mounted markers with respect to bones. Journal of 374 Biomechanics, 26, 864.

375 Bell, A.L., Brand, R.A., \& Pedersen, D.R. (1989). Prediction of hip-joint center location from external landmarks. Human Movement Science, 8, 3-16.

Besier, T.F., Lloyd, D.G., Cochrane, J.L. \& Ackland, T.R. (2001). Anticipatory effects on knee joint loading during running and cutting maneuvers. Medicine and Science in Sports and Exercise, 33, 1176-1181.

Bloomfield, J., Polman, R., \& O’Donoghue, P. (2007). Physical demands of different positions in FA premier league soccer. Journal of Sports Science and Medicine, 6, 63-70.

Boden, B.P., Dean, G.S., Feagin, J.A., \& Garrett, W.E. (2000). Mechanisms of anterior cruciate ligament injury. Orthopedics, 23, $573-578$.

Cappozzo, A., Cappello, A., Della Croce, U., \& Pensalfini, F. (1997). Surface marker cluster design for 3-D bone movement reconstruction' IEEE Transactions on Biomedical Engineering. 44, 1165-1174.

Cortes, N., Onate, J, \& van Lunen, B. (2011). Pivot task increases knee frontal plane loading compared with sidestep and drop-jump. Journal of Sports Sciences, 29, 83-92.

Dempsey, A.R., Lloyd, D.G., Elliot, B.C., Steele, J.R., Munro, B.J. \& Russo, K.A. (2007).

390 The effect of technique change on knee loads during sidestep cutting. Medicine and Science in Sports and Exercise, 39, 1765-1773. 
Dempsey, A.R., Lloyd, D.G., Elliot, B.C., Steele, J.R., \& Munro, B.J. (2009). Changing sidestep cutting technique reduces knee valgus loading. American Journal of Sports Medicine, $37,2194-2200$.

Dempster, W.T. (1955). Space requirements of the seated operator: Geometrical, kinematic, and mechanical aspects of the body with special reference to the limbs. WADC Technical Report 55-159, Wright-Patterson Air Force Base, OH: Wright Air Development Centre.

Faude, O., Junge, A., Kindermann, W. \& Dvorak, J. (2005). Injuries in female soccer players. A prospective study in the German national league. American Journal of Sports Medicine, 33, 1694-1700.

Graham-Smith, P., Atkinson, L., Barlow, R. \& Jones, P. (2009). Braking characteristics and load distribution in $180^{\circ}$ turns. Conference Proceedings: The $5^{\text {th }}$ Annual UK Strength and Conditioning Association Conference, 5-7 June 2009, Wyboston Lakes, Bedfordshire.

Greig, M. (2009). The influence of soccer-specific activity on the kinematics of an agility sprint. European Journal of Sports Science, 9, 23-33.

Grood, E.S., \& Suntay, W.J. (1983). A joint coordinate system for the clinical description of three dimensional motions: application to the knee. Journal of Biomechanical Engineering, $105,136-144$.

Havens, K.L. \& Sigward, S.M. (2015). Cutting mechanics: Relation to performance and anterior cruciate ligament injury risk. Medicine and Science in Sports Exercise, 47, 818-824.

Jamison, S.T., Pan, X., \& Chaudhari, A.M.W. (2012). Knee moments during run-to-cut maneuvers are associated with lateral trunk positioning. Journal of Biomechanics, 45, 18811885. 

joint loads during cutting in female soccer players. Human Movement Science, 42, 203-211.

416 Kristianlunds, E., Faul, O., Bahr, R., Myklebust, G., \& Krosshaug, T. (2014). Sidestep cutting 417 techniques and knee abduction loading: implications for ACL prevention exercises. British Journal of Sports Medicine, 48, 779-783.

Manal, K., McClay, I., Stanhope, S., Richards, J., \& Galinat, B. (2000). Comparison of 420 surface mounted markers and attachment methods is estimating tibial rotations during walking: an in vivo study. Gait and Posture, 11, 38-45.

McLean, S.G., Huang, X., \& van den Bogert, A.J. (2005). Association between lower extremity posture at contact and peak knee valgus moment during sidestepping: Implications for ACL injury. Clinical Biomechanics, 20, 863-870. differences in proximal control of the knee joint. Sports Medicine, 41, 541-557.

Olsen, O.E., Myklebust, G., Engebretsen, L. \& Bahr, R. (2004). Injury mechanisms for anterior cruciate ligament injuries in team handball. A systematic video analysis. American Journal of Sports Medicine, 32, 1002-1012. moments on ACL strain during single-leg landing: A simulation study. Journal of Biomechanics, 42, 280-285. moments increase anterior cruciate ligament strain more than either alone. Medicine and 
436 Sigward, S.M., \& Powers, C.M. (2007). Loading characteristics of females exhibiting

437 excessive valgus moments during cutting. Clinical Biomechanics, 22, 827-833.

438 Vincent, W.J. (2005). Statistics in Kinesiology. Champaign, Illinois: Human Kinetics, 439 (Chapter 7).

440 Winter, D.A. (2009). Biomechanics and motor control of human movement. (4th ed.). New

441 York: Wiley, (Chapters 2 \& 5).

442 Yeadon, M.R., Kato, T., \& Kerwin, D.G. (1999). Measuring running speed using photocells.

443 Journal of Sports Sciences, 17, 249-257.

\section{FIGURE AND TABLE LEGENDS}

446 Figure 1. Plan view of the experimental set-up.

447 Figure 2. Scatter plots for the relationships between initial knee abduction angle (2a) and

448 initial foot progression angle (2b) with peak knee abduction moments.

449 Table 1. Mean (SD) technique variables and the relationships to peak knee abduction 450 moments during pivoting.

451 Table 2. Stepwise multiple regression of predictors of peak knee abduction moments during 452 pivoting. 
TABLE 1

\begin{tabular}{|c|c|c|c|}
\hline \multirow[t]{2}{*}{ Variable } & \multirow[t]{2}{*}{ Mean (SD) } & \multicolumn{2}{|c|}{$\begin{array}{l}\text { Relationships to knee } \\
\text { abduction moments }\end{array}$} \\
\hline & & $\mathrm{R}$ & $\mathrm{R}^{2}$ \\
\hline $\begin{array}{l}\text { Knee Abduction } \\
\text { Moments } \quad\left(\mathrm{Nm}^{\left.\mathrm{k} \mathrm{kg}^{-1}\right)}\right. \\
\text { during weight acceptance } \\
\text { of final contact }\end{array}$ & $1.24(0.41)$ & & \\
\hline $\begin{array}{l}\text { Initial Foot Progression } \\
\text { Angle at final contact }\left(^{\circ}\right)\end{array}$ & $18(18.4)$ & $0.49 *$ & $24 \%$ \\
\hline $\begin{array}{l}\text { Initial Pelvis Rotation } \\
\text { Angle at final contact }\left(^{\circ}\right)\end{array}$ & $52(14.1)$ & 0.32 & $9.9 \%$ \\
\hline $\begin{array}{l}\text { Initial knee abduction } \\
\text { angle at final contact }\left(^{\circ}\right)\end{array}$ & $-4(4.9)$ & $-0.49 *$ & $24 \%$ \\
\hline $\begin{array}{l}\text { Initial hip abduction } \\
\text { angle at final contact }\left(^{\circ}\right)\end{array}$ & $-20(6.9)$ & 0.06 & $>1 \%$ \\
\hline $\begin{array}{l}\text { Initial hip rotation angle } \\
\text { at final contact }\left(^{\circ}\right)\end{array}$ & $14(9.1)$ & -0.35 & $12.3 \%$ \\
\hline $\begin{array}{l}\text { Initial Trunk Flexion } \\
\text { Angle }\left(^{\circ}\right)\end{array}$ & $18(9.5)$ & -0.26 & $6.9 \%$ \\
\hline $\begin{array}{ll}\text { Initial Lateral } & \text { Trunk } \\
\text { Flexion Angle }\left(^{\circ}\right) & \end{array}$ & $-1.9(5.8)$ & 0.20 & $3.8 \%$ \\
\hline $\begin{array}{l}\text { Initial Hip Flexion Angle } \\
\left({ }^{\circ}\right)\end{array}$ & $45(13.5)$ & -0.1 & $1 \%$ \\
\hline $\begin{array}{l}\text { Initial Knee } \text { Flexion } \\
\text { Angle }\left(^{\circ}\right)\end{array}$ & $24(6.3)$ & -0.03 & $<1 \%$ \\
\hline
\end{tabular}




\begin{tabular}{|c|c|c|c|}
\hline Initial Ankle Angle $\left(^{\circ}\right)$ & $58(11.6)$ & -0.04 & $<1 \%$ \\
\hline Last step length (m) & $0.79(0.07)$ & 0.18 & $3.1 \%$ \\
\hline $\begin{array}{l}\text { Horizontal touchdown } \\
\text { distance }(m)\end{array}$ & $0.66(0.04)$ & 0.02 & $<1 \%$ \\
\hline $\begin{array}{l}\text { Peak Horizontal Braking } \\
\text { Force Ratio }\end{array}$ & $0.94(0.19)$ & 0.19 & $3.5 \%$ \\
\hline $\begin{array}{l}\text { Peak hip flexor moments } \\
\left(\mathrm{Nm} \cdot \mathrm{kg}^{-1}\right)\end{array}$ & $2.54(0.69)$ & $-0.39 * *$ & $15 \%$ \\
\hline $\begin{array}{l}\text { Peak knee flexor } \\
\text { moments }\left(\mathrm{Nm} \cdot \mathrm{kg}^{-1}\right)\end{array}$ & $2.07(0.34)$ & -0.17 & $3 \%$ \\
\hline \multicolumn{4}{|l|}{$* p=0.01$} \\
\hline$* * \mathbf{p}<0.05$ & & & \\
\hline
\end{tabular}

462

463

464

TABLE 2

\begin{tabular}{|l|c|c|c|}
\hline Blocks & B & Standard errors $\beta$ & $\beta$ \\
\hline Block 1: & -0.03 & 0.015 & $-0.363^{*}$ \\
\hline Initial Knee Abduction Angle & & & \\
\hline Block 2: & 0.008 & 0.004 & $0.362^{*}$ \\
\hline Initial Foot Progression Angle & & & \\
\hline & $* \mathrm{p}<0.05$ & & \\
\end{tabular}

466

467 


\section{FIGURE 1}

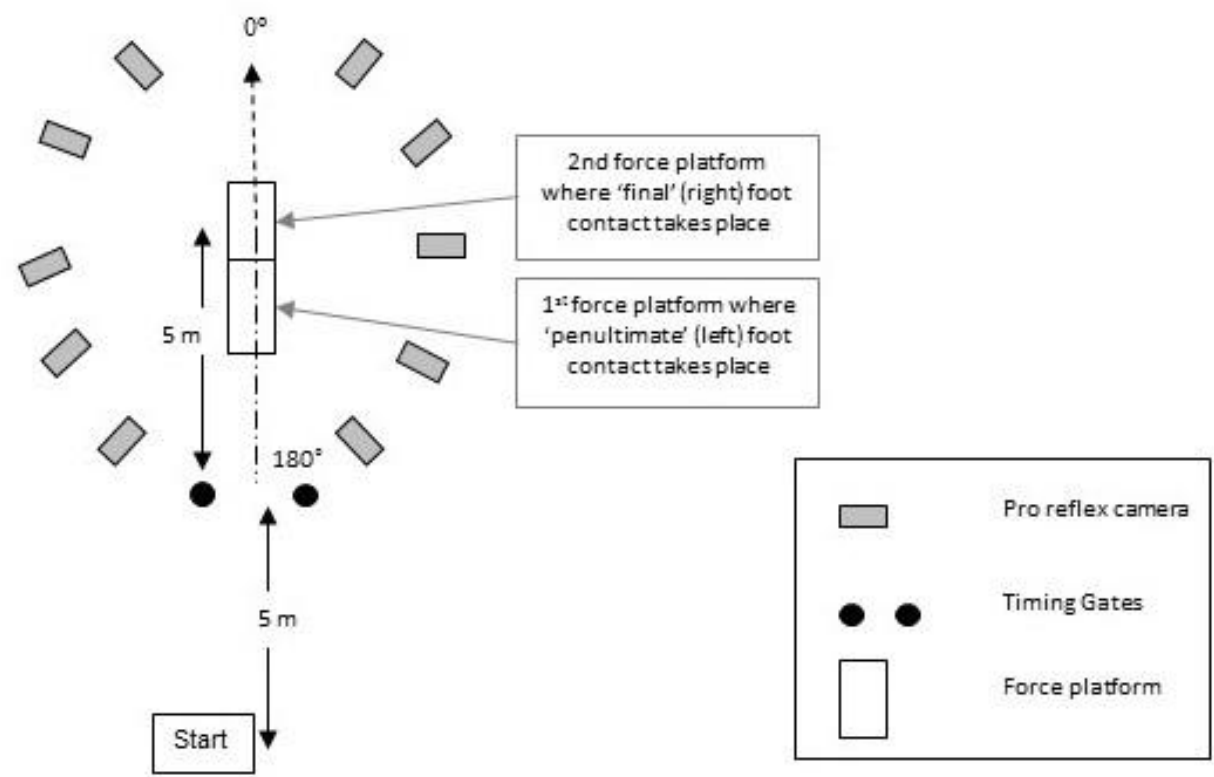


FIGURE 2

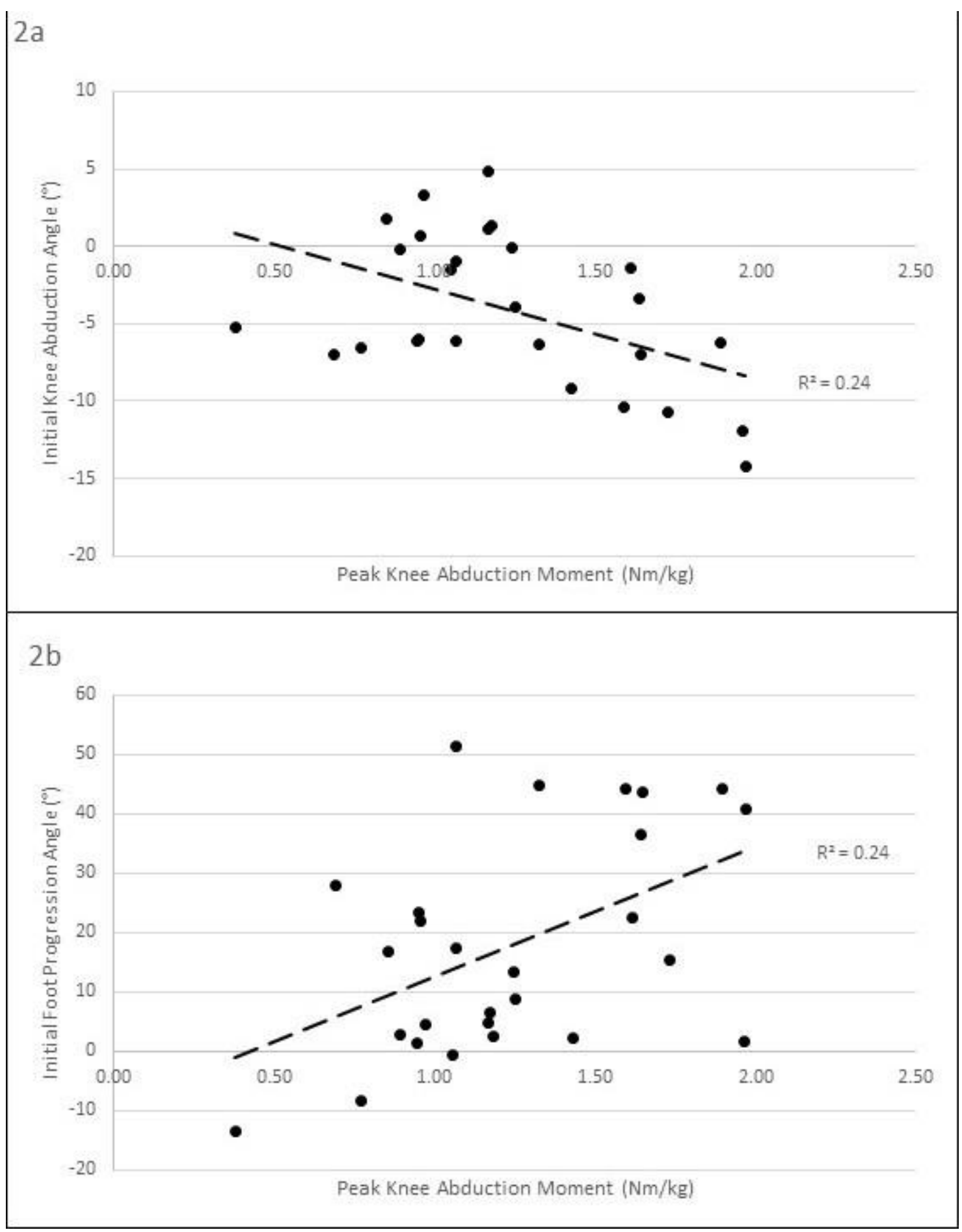

